characters are most akin to the shrubby Chinese species. In Europe and the outlier in North Africa, groups of allied species and varieties occupy the same geographical area, and in each of these groups one species is diploid with a much more restricted range than the others, which are tetraploid. Excluding the representatives in the Caucasus and Asia Minor, the Asiatic groups of species and varieties (which contain what are probably the most primitive species, including herbaceous members with more than one flower to a stem and also shrubby species) are all diploid, with the exception of one group which has a diploid species in Japan and a connected tetraploid on the Chinese mainland. Why in Asia and America do the diploid species so greatly outnumber the tetraploids, and why do tetraploids predominate in Europe? Colonel Stern suggests that events during and after the glacial period may have determined the cytological constitution of species and so influenced their distributional potential.

In Europe, by the southerly displacement of the vegetational zones during glacial times, the existing diploid peonies were forced into the Mediterranean basin and on to some of the Mediterranean islands. It is supposed that as a result of this geographical disturbance tetraploid species were derived from the diploids and, when conditions ameliorated, the more vigorous and better adapted tetraploids spread rapidly northwards and also crossed into north Africa. This hypothesis may explain why, in the European groups of related species occurring in the same areas, the diploid species have a much more restricted range than the derivative tetraploids. The diploid peonies of eastern Asia, it is surmised, are ancient relicts, which survived in situ during the glacial epoch and thus avoided genetic disturbance leading to tetraploidy. Cytological investigation of certain related genera and species may assist in the interpretation of their geographical range; but the genetic factor, while obviously important, must not be emphasized to the exclusion of other considerations. It seems significant that the genera in the British flora notorious for the numbers of 'critical' or endemic species have all been disrupted to some extent in the glacial periods, and the complications in these groups may be due to genetic disturbance resulting from climatic upheavals.

The individual species and varieties are fully described and discussed in Chapter 3 ; a full synonymy is given and specimens examined are cited. Unfortunately, scant mention is made of the collections, including types, preserved in the British Museum, and this disregard has resulted occasionally in an inadequate appreciation of the geographical range. For example, $P$. Delavayi, according to the monograph, is confined to Yunnan; but there is a specimen (Kingdon Ward 12214) from the Po-Yigrong region of south-east Tibet which was named by the author and yet is omitted from his enumeration. A more serious oversight has led to the treatment of Pallas's $P$. hybrida as a species non satis nota. Type-material is, however, preserved in the British Museum and, by Colonel Stern's classification, is referable to $P$. anomala var. intermedia.

One would have expected, in a book bearing the imprimatur of the Royal Horticultural Society, that some attempt would be made to deal with the hosts of named hybrids and forms which are more generally cultivated than the wild species; but for information on these, gardeners must still refer to nurserymen's catalogues. Apart from these criticisms, the enumera. tion reveals a careful and well-considered survey of the genus.

A fully documented chronological bibliography of the genus occupies Chapter 4, and a history of the species in cultivation with practical advice on their culture concludes the volume. The list of peony species and synonyms forming Appendix 1 gives no references to the text and could have been conveniently included in the general index.

"A Study of the Genus Pæonia" is a credit to the author, artists, printers and publishers, and sets a high standard in botanical and horticultural writing. The book is a splendid and timely token of British scholarship and eraftsmanship, and the freshness of the beautiful coloured plates calls for a special word of praise. Perhaps it is not too much to hope that the Society will sponsor further volumes in this style; they could scarcely fail to appeal to botanists and gardeners alike.

GEORgE TAYLOR

\section{TECHNICAL MATHEMATICS}

Applied Mathematics for Engineers and Physicists By Dr. Louis A. Pipes. Pp. xiii+618. (New York and London: McGraw-Hill Book Co., Inc., 1946.)$27 s .6 d$.

$\mathrm{T}$ HIS text is an excellent addition to the increasing number of books which seek to acquaint the physicist and engineer with the modern methods of mathematics as applied to technical problems.

The subject-matter is based on lectures given by the author at the Graduate School of Engineering, Harvard University, and covers both basic mathematics and relevant applications to physical and engineering problems such as conduction of heat and vibrations of beams.

Though general in scope, the book should be valuable to the specialist as a work of reference which can be consulted to ascertain the power and field of application of particular methods such as the use of matrices or of the operational calculus. Having chosen a method promising for his particular problem, the research worker can then proceed, it necessary, to a specialist book, references to which are given by Dr. Pipes at the end of each chapter.

On a point of detail, it is noted that two lists of Laplace transforms are given in widely separated chapters, involving considerable duplication, the first list being in excellent tabular form, whereas the second is given in the less easily consulted form of consecutive equations without tabular division. In future editions it is suggested that these lists could be amalgamated into one table at the end of the book for ease of reference. Apart from this minor criticism, the text appears well written and arranged and includes problems for the reader to test his skill or the power of a method.

Though primarily designed for the graduate, this text could well take a place on the bookshelf of the brighter undergraduate, since the subject-matter is unusually wide in range, covering, for example, both a first introduction to complex numbers in the second chapter and the analysis of non-linear oscillatory systems in the final chapter.

In general, this book can be recommended to all engineers and physicists interested in improving the range and power of the mathematical tools at their command.
E. N. Fox 